\title{
In the Basis of Hashimoto's Thyroiditis, to Form Papillary Thyroid Carcinoma, Metastasized and Then to De-Differentiate into Poorly Differentiated Squamous Cell Carcinoma
}

\author{
Xinle Ren ${ }^{1 *}$, Di Zhu ${ }^{1 *}$, Hen Wang1, Jilin Wang², Biyun Lin' ${ }^{1}$, Yongfang Ou${ }^{1}$, Bing Huang1, \\ Jian Huang1\# \\ ${ }^{1}$ The Center of Pathological Diagnosis and Research, Affiliated Hospital, Guangdong Medical University, Zhanjiang, China \\ ${ }^{2}$ Department of Pathology, Zhanjiang Central People's Hospital, Zhanjiang, China \\ Email: "18665763598@163.com
}

How to cite this paper: Ren, X.L., Zhu, D., Wang, H., Wang, J.L., Lin, B.Y., Ou, Y.F., Huang, B. and Huang, J. (2021) In the Basis of Hashimoto's Thyroiditis, to Form Papillary Thyroid Carcinoma, Metastasized and Then to De-Differentiate into Poorly Differentiated Squamous Cell Carcinoma. Journal of Cancer Therapy, 12, 254-267.

https://doi.org/10.4236/jct.2021.125024

Received: April 12, 2021

Accepted: May 16, 2021

Published: May 19, 2021

Copyright $\odot 2021$ by author(s) and Scientific Research Publishing Inc. This work is licensed under the Creative Commons Attribution International License (CC BY 4.0).

http://creativecommons.org/licenses/by/4.0/ (c) (i) Open Access

\begin{abstract}
Thyroid squamous cell carcinoma is very rare. At present, it is limited to case reports. Since the thyroid follicular epithelium is the non-squamous epithelium, how primary squamous cell carcinoma (SCC) of the thyroid occurs is still a controversial issue. Hashimoto's thyroiditis (HT) is considered to be an independent risk factor for thyroid cancer, under the basis of HT, how tumor cells evolve and develop to papillary thyroid carcinoma (PTC), and particularly to de-differentiate into SCC is elusive. We report a 72-year-old female patient who developed multiple subtypes of PTC on a basis of HT, and finally to de-differentiate into SCC within the local foci of lymph node metastasis. We found that there was a variety of sub-types of PTC in this patient in the background of HT. SCC was found within local lymph node metastasis. Pathomorphology, immunohistochemistry, and molecular pathology have confirmed that the SCC was derived from PTC, and then developed into poorly differentiated SCC and/or anaplastic carcinoma. We also conducted a comprehensive literature review.
\end{abstract}

\section{Keywords}

Papillary Thyroid Cancer, Thyroid Squamous Cell Carcinoma, Pathomorphology, Molecular Pathology, Multidisciplinary 


\section{Introduction}

Papillary thyroid carcinoma (PTC) is a relatively well-differentiated malignant tumor of the thyroid gland, accounting for more than $85 \%$ of all types of thyroid cancer [1]. The relationship between immunity and inflammation has been concerned by scientists for a long time. More and more evidence shows that the thyroid is an autoimmune organ, which can occur in a variety of autoimmunerelated inflammatory diseases, such as Hashimoto's thyroiditis (HT), chronic lymphocytic thyroiditis (CLT), and so on [2]. When HT occurs, in addition to a large number of interstitial lymphocyte infiltration and the formation of lymphoid follicles, fibrosis and calcification can also be appeared, especially the proliferation of thyroid follicular epithelium, and even form neoplastic hyperplasia. Up to $40 \%$ of PTC is accompanied by HT and/or CLT. On the basis of immune inflammation, thyroid cancer, especially PTC could be induced [3] [4] [5] [6]. However, the association between thyroid cancer and concomitant autoimmune thyroiditis is controversial [4] [7].

In addition to conventional PTC, there are other variants, including papillary microcarcinoma, follicular variant, tall cell variant, cribriform-morular variant, columnar cell variant, hobnail variant, solid/trabecular variant, warthin-like variant, oncocytic variant, et al. [8] [9]. Most of the PTCs are usually a single histological subtype, and a few cases can also have two or more histological subtypes [1] [10] [11]. More than $95 \%$ of thyroid malignant tumors are derived from thyroid follicular epithelium, which includes PTC, follicular thyroid cancer (FTC), poorly differentiated thyroid cancer (PDTC), and undifferentiated or anaplastic thyroid cancer (ATC), among them, PTC accounts for about 85\% [12] [13]. SCC accounts for only $0.1 \%-1 \%$ of thyroid cancer [13] [14]. In theory, the thyroid gland has no squamous epithelium, and how primary squamous cell carcinoma of the thyroid occurs is still a controversial issue. At present, some researchers believe that thyroid SCC may come from embryonic residues [15]. The literature on thyroid SCC is limited to case reports [14] [16] [17] [18] [19] [20]. Whether thyroid SCC can become cancerous on the basis of HT, and then form from PTC to squamous metaplasia is worthy of further discussion. Due to the different clinical prognosis and treatment strategies of different types of thyroid cancer, especially thyroid SCC, it is necessary to make an in-depth study of this kind of cases.

Here, we present a special case of PTC with various subtypes in the background of HT and transforming into SCC in local lymph node metastases. A comprehensive literature review was also carried out.

\section{Materials and Methods}

\subsection{Patient}

A 72-year-old female underwent bilateral total thyroidectomy plus left cervical lymph node dissection due to thyroid cancer in the local hospital, one and a half years ago. The pathological diagnosis was PTC on the left side of the thyroid, and was a nodular goiter on the right side of the low thyroid lobe. The patient took le- 
vothyroxine sodium tablets 100 micrograms per day after the operation. She denies having a medical history of high blood pressure, diabetes, and other diseases.

Half a year ago, she was found a new mass in the left neck in our hospital. Ultrasounds showed that the mass about $2.5 \mathrm{~cm}$ in diameter was on the left side of the neck. PET-CT showed that there was a mass in area II-V of the left neck, which showed necrosis and increased glucose metabolism, and lymph node metastasis was considered. Needle biopsy showed lymph node metastatic SCC in pathology on the left side of the neck lymph node. Ear, nose, and throat (ENT) evaluation with fiberoptic transnasal laryngoscopy was negative for other head and neck primary tumors. Then, a cleaning operation for lymph nodes on the left side was performed.

\subsection{Pathological Examination}

Specimen handling: The postoperative specimens were carefully observed to describe the size, color, texture, and other characteristics of the mass. The processes of sampling, dehydration, embedded in paraffin, cut slides, and hematoxylin and eosin (H\&E) staining were performed according to the requirements.

Immunohistochemical staining (IHC): We used 18 different related antibodies to conduct an IHC comparative study of primary PTC and SCC in lymph node metastasis. The operation of IHC is by the guidelines of the Dako Omnis machine. All 18 antibodies including clones, manufacturer, and dilutions were showed Table 1 .

Table 1. Antibody clones, manufacturer, and dilutions used in the immunohistochemical study.

\begin{tabular}{|c|c|c|c|c|c|c|c|}
\hline Antibody & Gene symbol & Clone & Species & Vendor & Address & Cat\# & Dilution \\
\hline p53 & TP53 & DO-7 & Mouse monoclonal & Ventana & Tucson, AZ & 800-2912(G04027) & Prediluted \\
\hline CD56 & NCAM1 & MX039 & Mouse monoclonal & MXB Biotechnologies & Fuzhou, China & MAB-0743(2007090743C4) & $1: 150$ \\
\hline CK5/6 & KRT5/KRT6 & MX040 & Mouse monoclonal & DAKO & Carpinteria, CA & IR780(20077790) & $1: 150$ \\
\hline CK19 & KRT19 & A53B/A2.26 & Mouse monoclonal & MXB Biotechnologies & Fuzhou, China & MAB-0829(2007300056C3) & $1: 75$ \\
\hline Ki67 & MKI67 & MIB-1 & Mouse monoclonal & DAKO & Carpinteria, CA & IR626(20081075) & Prediluted \\
\hline $\mathrm{P} 40$ & TP63 & ZR8 & Mouse monoclonal & MXB Biotechnologies & Fuzhou, China & RMA-0815(2007300815C4) & $1: 150$ \\
\hline P63 & TP63 & DAK-P63 & Mouse monoclonal & DAKO & Carpinteria, CA & IR662(20081031) & Prediluted \\
\hline TG & TG & $2 \mathrm{H} 11+6 \mathrm{E} 1$ & Mouse monoclonal & MXB Biotechnologies & Fuzhou, China & MAB-0797(1908150797C2) & Prediluted \\
\hline TPO & TPO & $2 \mathrm{G} 2$ & Mouse monoclonal & MXB Biotechnologies & Fuzhou, China & MAB-0800(2007300800C3) & $1: 150$ \\
\hline PAX-8 & PAX-8 & EP298 & Rabbit monoclonal & MXB Biotechnologies & Fuzhou, China & RMA-0817(20073000817C2) & Prediluted \\
\hline b-Cantenin & CTNNB & MX043 & Mouse monoclonal & MXB Biotechnologies & Fuzhou, China & MAB-0754(1904080754C2) & Prediluted \\
\hline TTF-1 & TTF-1 & MX011 & Mouse monoclonal & MXB Biotechnologies & Fuzhou, China & MAB-0599(2007090599C4) & $1: 150$ \\
\hline PD-L1 & CD274 & SP263 & Rabbit monoclonal & Ventana & Tucson, AZ & 743-7066(G13629) & Prediluted \\
\hline BRAF-V600E & BRAF & VE1 & Mouse monoclonal & Ventana & Tucson, AZ & 790-5095(G01373) & Prediluted \\
\hline $\mathrm{CT}$ & CALCA & SP17 & Rabbit monoclonal & MXB Biotechnologies & Fuzhou, China & RMA-0553(181101553C2) & Prediluted \\
\hline Gal-3 & LGALS3 & $9 \mathrm{C} 4$ & Mouse monoclonal & MXB Biotechnologies & Fuzhou, China & MAB-0572(1904080572C3) & Prediluted \\
\hline MC & $\mathrm{N} / \mathrm{A}$ & HBME-1 & Mouse monoclonal & MXB Biotechnologies & Fuzhou, China & MAB-0130(1912050130C4) & $1: 50$ \\
\hline SYN & SYP & MX038 & Rabbit monoclonal & MXB Biotechnologies & Fuzhou, China & MAB-0742(2007020742C1) & $1: 70$ \\
\hline
\end{tabular}


Q-PCR: The BRAF exon 15, KRAS exon 2-4, NRAS exon 2-4, HRAS exon 3, PIK3CA exon 20, and TERT promoter mutation in the primary PTC foci and metastatic SCC foci were detected by the method of fluorescence PCR using the human gene mutation detection kit made from Amoy Dx Biology Co., Ltd. The operation process is based on the manufacturer's instructions.

\section{Results}

\subsection{Morphological Examination}

Grossing, a piece of gray-red tissue $5 \times 3 \times 2 \mathrm{~cm}$ in volume was removed from the left thyroid. The section showed a mass of $2.5 \mathrm{~cm}$ in diameter, gray-white colors, and hard in texture. The resected right thyroid gland was $4 \times 2.5 \times 2 \mathrm{~cm}$ in volume, with grayish red colors, nodular shape, and medium texture. Besides, more than 20 lymph nodes in the central region of the left neck and 6 - 8 cervical regions were examined. The lymph nodes are enlarged, ranging from 0.5 to 3 centimeters in diameter. Histologically, on the left side of the thyroid, H\&E slides showed hyperplasia of thyroid follicular epithelial under the background of HT (Figure 1(a)), and the follicular epithelium in the focal area of HT was transformed into PTC (Figure 1(b)). Importantly, in addition to a typical subtype of PTC (Figure 1(c)), there were other subtypes of PTC, such as oncocytic variant (Figure 1(d)), follicular and solid patterns (Figure 1(e)). In some focal areas, we also found metaplasia of columnar epithelium into squamous epithelium (Figure 1(f)).

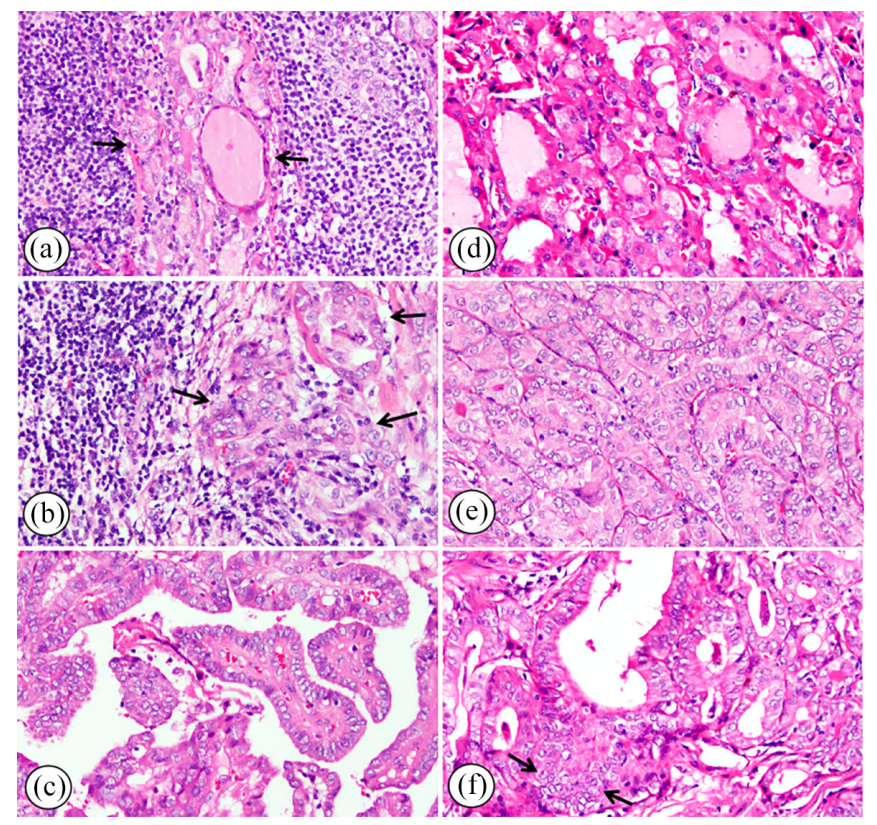

Figure 1. H\&E staining showed different histological subtypes of PTC in primary tumors in the background disease of HT. Thyroid follicular epithelial hyperplasia in the background of HT shown by the black arrow (a); Carcinogenesis of thyroid follicular epithelium in the background of HT shown by the black arrow (b); Classical PTC (c); Oncocytic PCT (d); Solid and follicular PTC (e); Local squamous metaplasia in tall cell columnar subtype of PTC shown by the black arrow (f). Magnification 200×. 


\subsection{Immunohistochemistry Staining (IHC)}

To further clarify the nature and types of the tumor, we performed IHC on a series of antibodies, including CK19, TPO, TG, CD56, TTF-1, Galectin-3, etc. on formalin-fixed paraffin-embedded (FFPE) tissues. A list of all antibodies is shown in Table 1. IHC indicated that it was a typical pattern of PTC in which CK19, TTF1, and Galectin-3 were strong positive, and TPO, CD56, and TG were negative showed in Figures 2(a)-(f). IHC also showed that CT, MC, and Syn were negative (not showed).

\subsection{Imaging and Surgical Findings}

After the first operation, the patient took levothyroxin sodium tables 100ug/day regularly. Fourteen months later, the patient herself found a mass in the anterior region of the left neck, which was gradually enlarged, and the left upper limb occasionally felt numbness and pain, and then came to our hospital for a medical treatment. Physical examination: A mass was palpable in the anterior region of the left neck, about $8 \mathrm{~cm}$ in maximum diameter, texture hard, unclear boundary, and poor mobility. Contrast-enhanced CT scanning in the neck diagnosed that the thyroid gland in both sides was absent, and the mass in the left neck supraclavicular region was considered as PCT postoperative recurrence or lymph nodes metastasis (Figure 3(a)). Systemic PET/CT examination: PET/CT showed that the bilateral lobe of the thyroid was absent after operation, and there was no

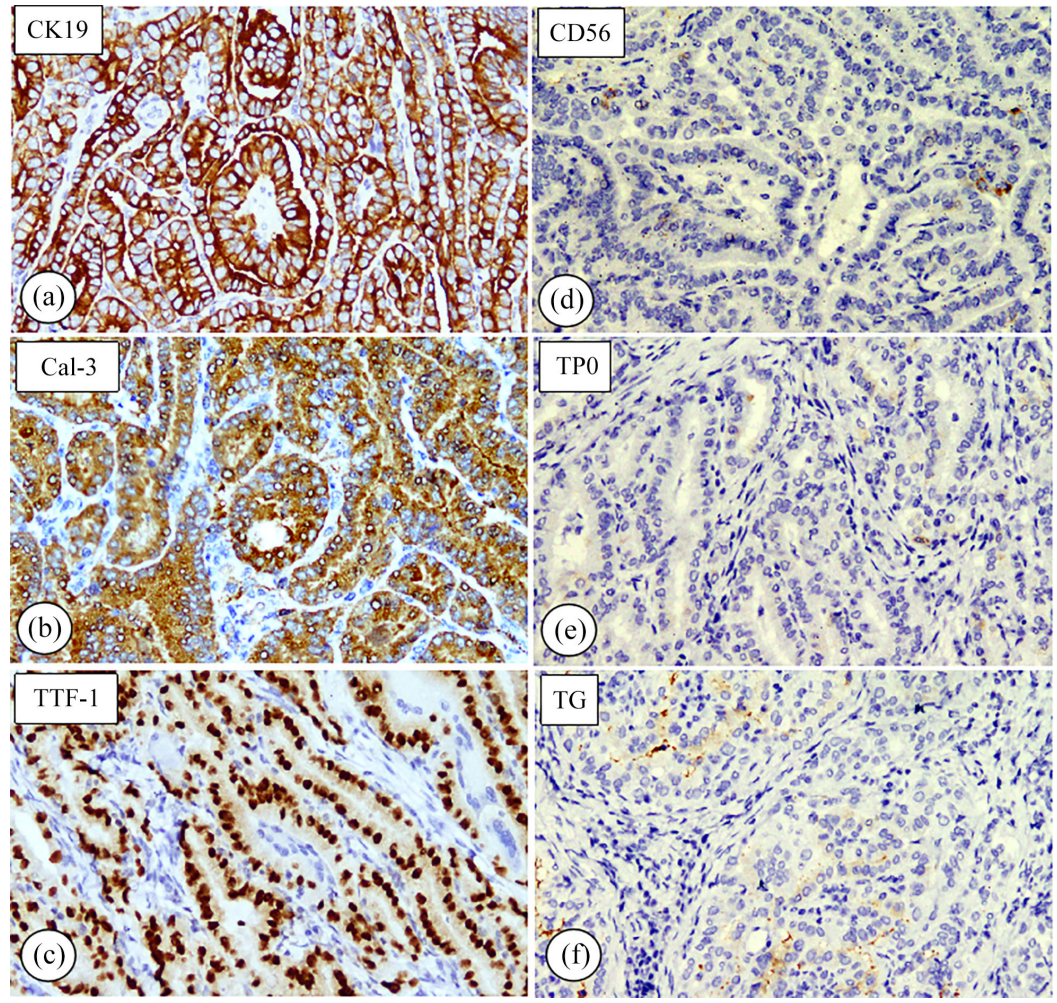

Figure 2. IHC showed that CK19, Gal-3, TTF-1, CD56, TPO and TG were expressed in primary PTC. CK19, Gal-3 and TTF-1 were positive (a)-(c); CD56, TPO and TG were negative (d)-(f). Magnification 200x. 

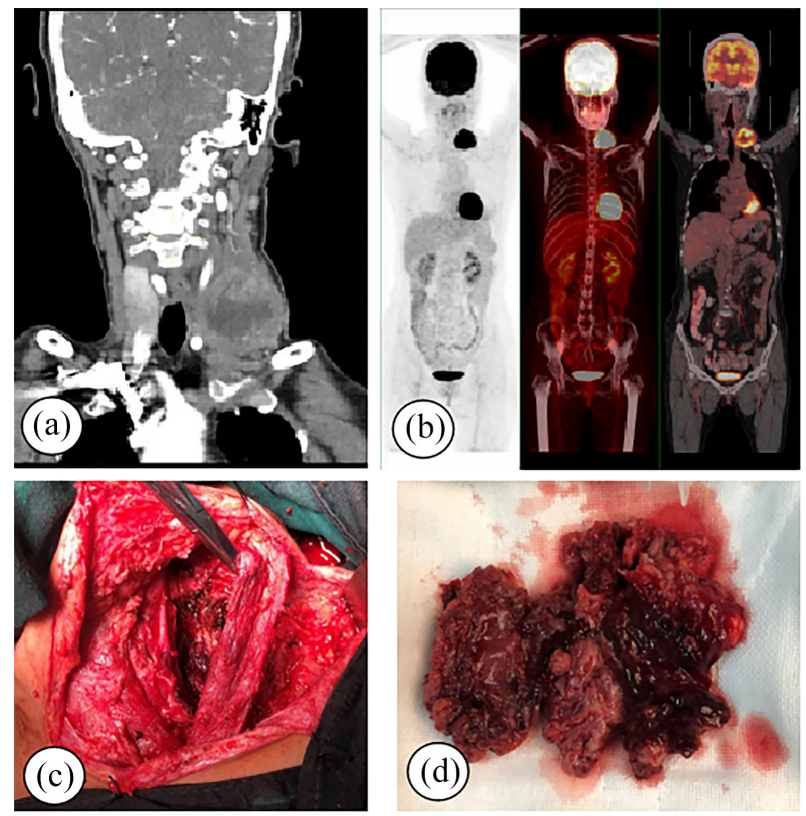

Figure 3. Imaging and surgical findings. Contrast-enhanced CT scanning indicated a mass in the left neck supraclavicular region (a); PET/CT showed a mass in the IV-V area of the left neck showed internal necrosis and increased glucose metabolism, considering a metastatic tumor (b); Showing the surgical area of the left neck (c); Showing multiple lymph nodes were removed in the left neck II-V regions (d).

definite sign of a malignant tumor in the operation area. The tumor in the IV-V area of the left neck showed internal necrosis and increased glucose metabolism in a ring. There were more then 20 lymph nodes metastases showed in the left neck (II-V) region showed in Figure 3(b).

After multidisciplinary discussion in our hospital, it was considered that there was a symptom of tumor compression, and due to the effect of radiotherapy and chemotherapy was not obvious, so we choose the operation to reduce the burden of the tumor. It was found during the operation that the boundary among the original operating area and sternocleidomastoid muscle, and local cervical skin was not clear. The space between the lesion and the common carotid artery was disappeared, the trachea was compressed to the right, and the left internal jugular vein was not seen (showed in Figure 3(c)). Multiple lymph nodes were removed in the left neck II-V regions, it was possible to consider lymph nodes metastasis (showed in Figure 3(d)).

\subsection{Pathological Examination of Local Lymph Nodes}

Postoperative pathology confirmed that it was metastatic carcinoma of lymph nodes. Interestingly, there were various types of carcinoma in metastatic lesions, including classical papillary carcinoma (Figure 4(a)), tall columnar papillary carcinoma (Figure 4(b)), SCC (Figure 4(c)), and undifferentiated cell carcinoma or ATC (Figure 4(d)). In order to further clarify the characteristics of these tumor cells, we performed IHC on these tumor cells. There was a patchy nesting area of poorly differentiated or undifferentiated carcinoma in local lymph node 
metastases (Figure 5(a)). The IHC results showed that the tumor cells had the dual characteristics of PTC and SCC, which P40, P63, CK5/6, TTF-1, and CK19 were strongly positive showed in figures $5 \mathrm{~B}-\mathrm{F}$. These results strongly suggested that the tumor had double characteristics of PTC and SCC.

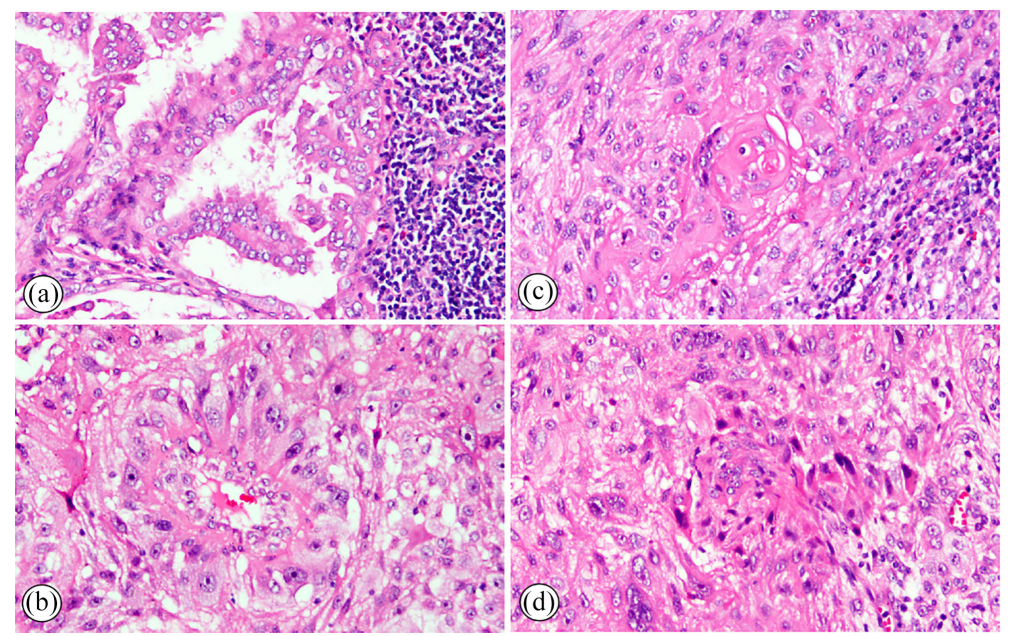

Figure 4. Different histological sub-types of thyroid carcinoma were shown in lymph node metastases. Classical PTC, The papillae tend to be thin with well-formed fibrovascular cores showing various degrees of arborization (a); Tall columnar PTC (b); Highly differentiated SCC (c); Undifferentiated carcinoma or thyroid anaplastic cancer (d). Magnification 200x.

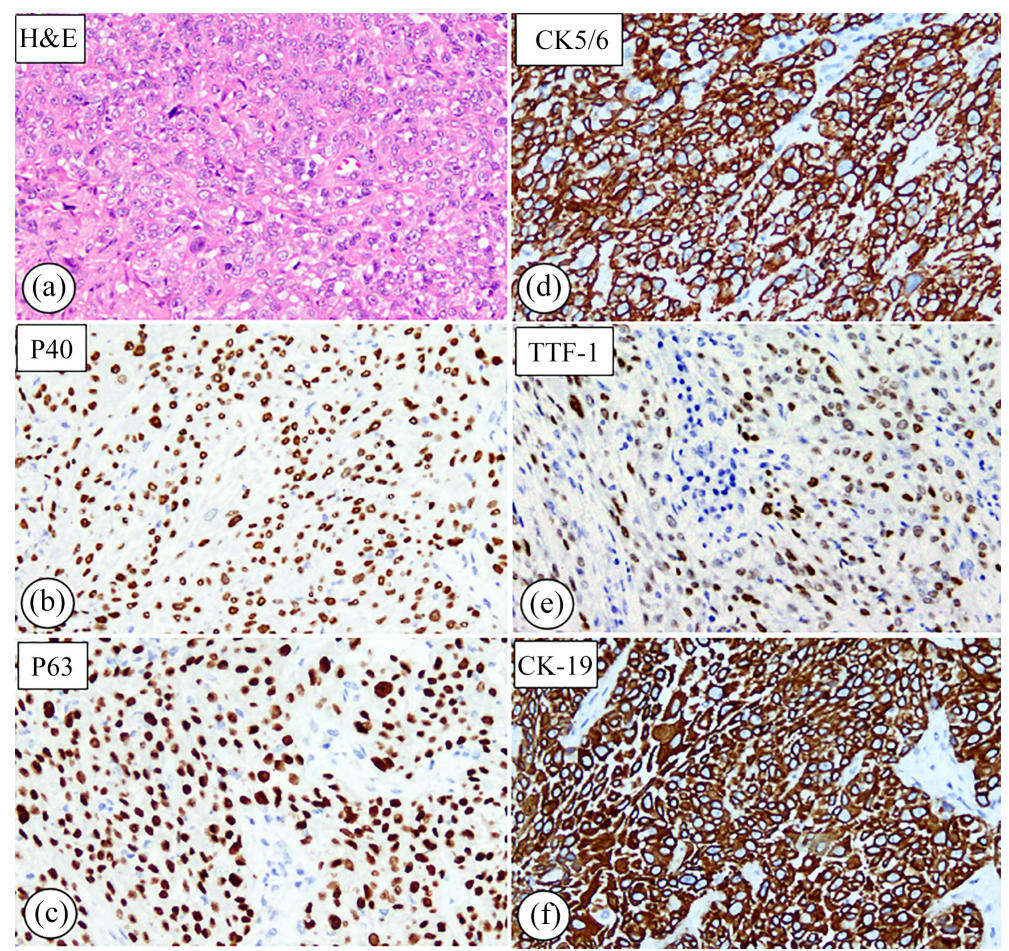

Figure 5. IHC showed the tumor cells had a double characteristics of PTC and SCC. H\&E staining showed the area of undifferentiated carcinoma in local lymph node metastases (a); P40, P63, CK5/6, TTF-1 and CK19 were positive (b)-(f). 


\subsection{A comparative Study of IHC and Molecular Pathology in Primary PTC and SCC within Local Lymph Node Metastasis}

For further exploring the biological and origin relationship between primary PTC and SCC in local lymph node metastasis, we made a comparative study by IHC and molecular pathology. Firstly, we compared the expression of PD-L1, TP53, $\mathrm{BRAF}^{\mathrm{V} 600 \mathrm{E}}$, and Ki67 in primary thyroid tumor and local lymph node metastasis by IHC. The results indicated that PD-L1 is negative in the primary tumor (Figure 6(a)) and a strong positive in metastatic tumors (Figure 6(e)). TP53 was expressed in both primary and metastatic tumors, but the positive rate in tumor cells was different. The expression rate of TP53 in primary tumors is about 20\% (Figure 6(b)), while in metastatic tumors, the expression rate of TP53 is about $80 \%$ (Figure 6(f)). BRAF ${ }^{\mathrm{V} 600 \mathrm{E}}$ was expressed in both primary and metastatic tumors, and there was no significant difference in the positive rate (Figure 6(c) and Figure 6(g))). In primary tumors, the Ki67 proliferation index is about $5 \%$ (Figure 6(d)), while in metastatic tumors, the Ki67 proliferation index is about $30 \%$ (Figure $6(\mathrm{~h})$ ). Then, we detected the mutations of BRAF exon 15, KRAS exon 2-4, NRAS exon 2-4, HRAS exon 3, PIK3CA exon 20, and TERT promoter mutation in primary and metastatic tumors by QPCR to further clarify the relationship between them. The results showed that BRAF exon 15 mutation was found in both primary and metastatic tumors, and no other gene mutations were found.

\subsection{Further Treatment and Follow-up}

After the second operation, she has been received radiotherapy for several months. There was no recurrence of local tumor and the patient is still under follow-up.

\section{Discussion}

This is a complicated case which is in the basis of HT, to induce into PTC, and form a variety of subtypes and different stages of PTC. The tall-cell subtype of

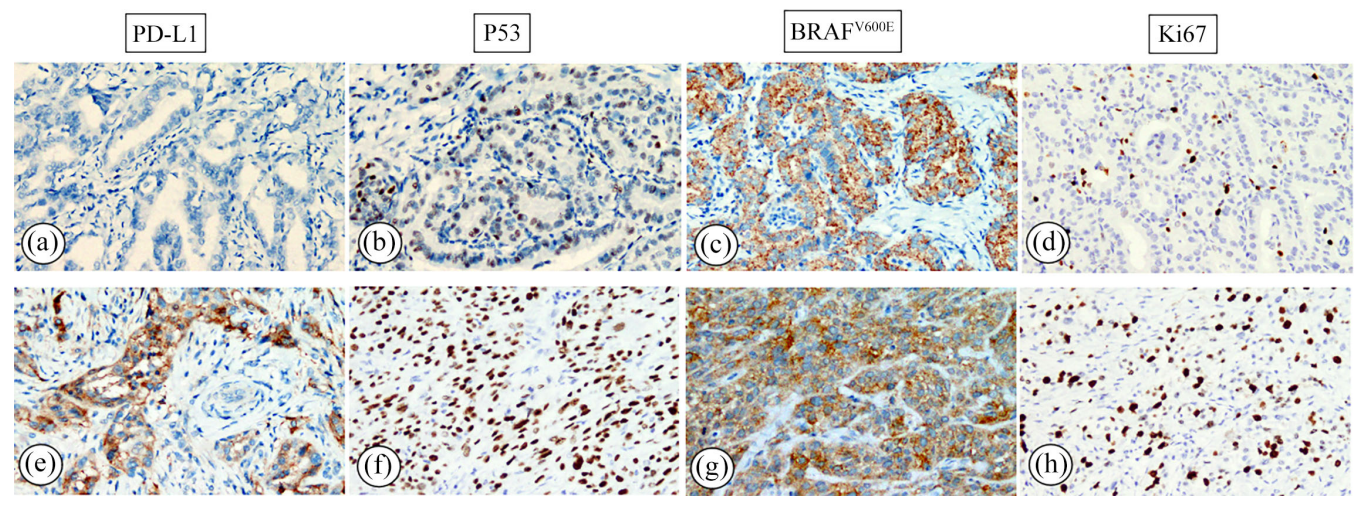

Figure 6. A comparative study of IHC in primary PTC and SCC in local lymph node metastasis. PD-L1 is negative in primary tumor (a) and strong positive in metastatic tumors (e); TP53 is about $20 \%$ in primary tumors (b) and is about $80 \%$ in metastatic tumors (f); $\mathrm{BRAF}^{\mathrm{V} 600 \mathrm{E}}$ was expressed in both primary (c) and metastatic tumors (g), and there was no significant difference in the positive rate; Ki67 is about $5 \%$ in primary tumor $(\mathrm{d})$, is about $30 \%$ in metastatic tumors (h). 
PTC further metaplasia into squamous cell carcinoma, and de-differentiate into undifferentiated carcinoma.

The association between thyroid autoimmune inflammatory disorders and cancer has attracted much attention for a long time. Changes in autoimmune inflammatory diseases, such as HT and CLT, can be coexisted in about $40 \%$ of thyroid cancers, especially PTC. This has to make people associate autoimmune inflammation of the thyroid gland with thyroid cancer. HT is the most common thyroid immune diseases. There is overwhelming evidence to show that HT is closely related to thyroid cancer, especially PCT [2]-[7], [21] [22] [23]. Giuseppa G. et al. found that up to $40.2 \%$ of the patients coexisted both diffuse HT and PTC in a study of 305 cases of PTC [4]. Several other studies have also shown that HT is closely related to PTC [2] [5] [21] [22]. Studies also suggested that HT might be an independent risk factor for thyroid cancer [23]. It seems like that the factor of HT may promote the development of thyroid cancer. The case we provided here showed that the atypical hyperplasia and canceration of the focal thyroid follicular epithelium were commonly noted in the background of HT. Our case provides further evidence that there is a close relationship between HT and thyroid cancer. On the other hand, HT might decrease the stages of differentiated thyroid cancer, and had a good prognosis with HT [7]. Therefore, it is most likely that thyroid autoimmune inflammation has different effects on the occurrence, development, and prognosis of different types and stages of thyroid cancer. The possible mechanism of thyroid cancer caused by thyroid autoimmune inflammation remains to be further studied.

The appearance of different subtypes of PTC in the same tumor is a manifestation of tumor diversity, and its essence is determined by intratumoral heterogeneity (ITH), and ITH are determined by the diversity of tumor genetic background [24]. In our case, there are several different histological subtypes of PTC, such as classical, oncocytic, solid and follicular, and tall cell columnar subtypes. Different subtypes of histological types in the same tumor must have different genetic alterations and biological characteristics, and also have a different response to treatment. These subtypes also represent different differentiation stages and different prognosis of thyroid carcinoma. Therefore, in clinicopathological diagnosis, we need to label each different histological subtype. In addition to different subtypes of PTC, the morphology of ATC can also be seen in this case. Although there are many studies on the relationship between different histological types of thyroid cancer and prognosis, opinions are still controversial [25] [26]. It has been debated for a long time whether ATC arise de novo or derive from the evolution of a preexisting well-differentiated carcinoma through either "anaplastic transformation" or "dedifferentiation" driven by the gaining of genetic abnormalities [8]. The existence of poorly differentiated forms with morphological, prognostic, and genetic features intermediate between well-differentiated and anaplastic carcinomas seems to support the hypothesis of a multistep carcinogenic process. In these series, PTC represents the most common histotype associated with anaplastic carcinoma [10]. 
In addition to different subtypes of PTC, the most notable phenomenon was that squamous cell carcinoma or poor-differentiated ATC appear in lymph node metastases. What's most interesting was that these poor-differentiated tumor cells express double antigens of PTC and SCC, which P40, P63, CK5/6, TTF1 and CK19 were all strong positive. The results of molecular pathology also showed that $\mathrm{BRAF}^{\mathrm{V} 600 \mathrm{E}}$ mutations were found in both PTC in the primary tumor and SCC in lymph node metastasis. These findings indicated that PTC and SCC have a similar genetic background, and strongly suggest that SCC or poor-differentiated ATC may develop from PTC. So far, we have only seen one case reported regarding BRAF mutation (c.1799 T > A; 1801_1812del) in primary SCC of the thyroid [27]. As we know that BRAF mutations, especially, BRAF ${ }^{\mathrm{V} 600 \mathrm{E}}$ mutation is the most common molecular genetic change in PTC [28] [29] [30] [31] [32]. Therefore, we believe that the SCC in the metastatic foci of this case may be evolved from the tall-cell subtype of PTC.

Our understanding of the prognosis of different subtypes of PTC is still insufficient, and the biological characteristics of different subtypes of PTC are still controversial. Allen S.Ho et al. found that the prognosis of well-differentiated PTC (WDPTC), poorly differentiated cancer, and variant cancer is significantly different, and suggesting that treatment should be tailored to specific histologic subtypes [25]. Different histological subtypes of PTC have different invasiveness. For example, the tall-cell subtype is more aggressive than the classic type [11]. In general, different histopathological types of thyroid cancer will affect the prognosis of their patients. Therefore, a multidisciplinary care plan requires surgical pathologists to be aware of the "so-called" aggressive variants of PTC. It is recommended that the proportion of thyroid cancer of different histological subtypes should be noted in the pathological diagnosis report [11]. It is essential to distinguish different subtypes of PTC, because of different histological subtypes, the treatment and prognosis are also different [1] [33] [34]. Research indicated that the most invasive variants of PTC are: diffuse sclerosis variant (DSV), tall cell variant (TCV), columnar cell variant (CCV), solid variant (SV), and hobnail variant $(\mathrm{HV})$. These variants are generally closely related to higher recurrence and metastasis rates and have low sensitivity to chemotherapy and radioactive iodine therapy, and the survival rate may also be low [1] [25] [33] [35]-[41]. Finally, we compared the biological characteristics of tumor cells in primary thyroid carcinoma and lymph node metastasis. The results showed that the expression of Ki67 and TP53 in lymph node metastasis was much higher than that in primary lymph node metastasis, indicating that a more malignant tumor cell population appeared in lymph node metastasis, and further revealed the process of tumor progression. It was also found that PD-L1 changed from negative in the primary foci to positive in the lymph node metastasis. The significance of this change needs to be further studied. BRAF ${ }^{\mathrm{V} 600 \mathrm{E}}$ proteins were positive by IHC, and $\mathrm{BRAF}^{\mathrm{V} 600 \mathrm{E}}$ were mutated by molecular pathological test, in both primary thyroid carcinoma and lymph node metastasis. It is further suggested that their origins may be the same. 
This is a good example that shows a continuous process of developing into PTC based on HT, then transforming into SCC, developing into lymph node metastatic carcinoma, and further dedifferentiating into highly malignant anaplastic carcinoma. In particular, there are some tumor cell subsets with different histological subtypes and biological characteristics, which brings great difficulties to clinical diagnosis and treatment. Although the patient underwent two operations, plus radiotherapy, the effect of treatments was still not good due to the phenotypic transformation of highly malignant tumor cells. We also recognize once again that primary thyroid SCC are actually derived from the subtype of tall columnar cells of PTC, and can further evolve into undifferentiated carcinoma.

\section{Ethics Statement}

This case was reviewed and approved by the Ethics Committee of Affiliated Hospital, Guangdong Medical University, China. The patient provided her written informed consent to participate in this study.

\section{Conflicts of Interest}

The authors declare no conflicts of interest regarding the publication of this paper.

\section{References}

[1] Coca-Pelaz, A., Shah, J.P, Hernandez-Prera, J.C., Ghossein, R.A., Rodrigo, J.P., Hartl, D.M., et al. (2020) Papillary Thyroid Cancer-Aggressive Variants and Impact on Management: A Narrative Review. Advances in Therapy, 37, 3112-3128. https://doi.org/10.1007/s12325-020-01391-1

[2] Molnár, C., Molnár, S., Bedekovics, J., Mokánszki, A., Győry, F., Nagy, E., et al. (2019) Thyroid Carcinoma Coexisting with Hashimoto's Thyreoiditis: Clinicopathological and Molecular Characteristics Clue up Pathogenesis. Pathology \& Oncology Research, 25, 1191-1197. https://doi.org/10.1007/s12253-019-00580-w

[3] Ferrari, S.M., Fallahi, P., Elia, G., Ragusa, F., Ruffilli, I., Rosaria Paparo, S., et al. (2020) Thyroid Autoimmune Disorders and Cancer. Seminars in Cancer Biology, 64, 135-146. https://doi.org/10.1016/j.semcancer.2019.05.019

[4] Graceffa, G., Patrone, R., Vieni, S., Campanella, S., Calamia, S., Laise, I., et al. (2019) Association between Hashimoto's Thyroiditis and Papillary Thyroid Carcinoma: A Retrospective Analysis of 305 Patients. BMC Endocrine Disorders, 19, Article No. 26. https://doi.org/10.1186/s12902-019-0351-x

[5] Jackson, D., Handelsman, R.S., Farrá, J.C. and Lew, J.I. (2020) Increased Incidental Thyroid Cancer in Patients with Subclinical Chronic Lymphocytic Thyroiditis. Journal of Surgical Research, 245, 115-118. https://doi.org/10.1016/j.jss.2019.07.025

[6] Paparodis, R.D., Karvounis, E., Bantouna, D., Chourpiliadis, C., Chourpiliadi, H., Livadas, S., et al. (2020) Incidentally Discovered Papillary Thyroid Microcarcinomas Are More Frequently Found in Patients with Chronic Lymphocytic Thyroiditis than with Multinodular Goiter or Graves' Disease. Thyroid, 30, 531-535. https://doi.org/10.1089/thy.2019.0347

[7] Borowczyk, M., Janicki, A., Dworacki, G., Szczepanek-Parulska, E., Danieluk, M., 
Barnett, J., et al. (2019) Decreased Staging of Differentiated Thyroid Cancer in Patients with Chronic Lymphocytic Thyroiditis. Journal of Endocrinological Investigation, 42, 45-52. https://doi.org/10.1007/s40618-018-0882-4

[8] Orlp, L.R. (2017) WHO Classification of Tumours of Endocrine Organs. 4th Edition, France International Agency for Research on Cancer (IARC), Lyon.

[9] Bongiovanni, M., Sykiotis, G.P., La Rosa, S., Bisig, B., Trimech, M., Missiaglia, E., et al. (2020) Macrofollicular Variant of Follicular Thyroid Carcinoma: A Rare Underappreciated Pitfall in the Diagnosis of Thyroid Carcinoma. Thyroid, 30, 72-80. https://doi.org/10.1089/thy.2018.0607

[10] Ragazzi, M., Torricelli, F., Donati, B., Ciarrocchi, A., de Biase, D., Tallini, G., et al. (2020) Coexisting Well-Differentiated and Anaplastic Thyroid Carcinoma in the Same Primary Resection Specimen: Immunophenotypic and Genetic Comparison of the Two Components in a Consecutive Series of 13 Cases and a Review of the Literature. Virchows Archiv, 478, 265-281. https://doi.org/10.1007/s00428-020-02891-9

[11] Hernandez-Prera, J.C. (2020) The Evolving Concept of Aggressive Histological Variants of Differentiated Thyroid Cancer. Seminars in Diagnostic Pathology, 37, 228233. https://doi.org/10.1053/j.semdp.2020.03.002

[12] Dralle, H., Machens, A., Basa, J., Fatourechi, V., Franceschi, S., Hay, I.D., et al. (2015) Follicular Cell-Derived Thyroid Cancer. Nature Reviews Disease Primers, 1, Article No. 15077. https://doi.org/10.1038/nrdp.2015.77

[13] Limberg, J., Ullmann, T.M., Stefanova, D., Finnerty, B.M., Beninato, T., Fahey III, T.J., et al. (2020) Prognostic Characteristics of Primary Squamous Cell Carcinoma of the Thyroid: A National Cancer Database Analysis. World Journal of Surgery, 44, 348-355. https://doi.org/10.1007/s00268-019-05098-5

[14] Lim, A.E., Sooby, P. and Townsley, R.B. (2020) Primary Thyroid Squamous Cell Carcinoma-A Scottish National Case Series. Scottish Medical Journal, 65, 60-63. https://doi.org/10.1177/0036933020916348

[15] Puccini, M., Roffi, N., Pucci, V., Fiacchini, G., Ugolini, C. and Buccianti, P. (2020) Synchronous Squamous Cell Carcinoma and Papillary Thyroid Carcinoma Arising from the Thyroglossal Duct Remnant: Case Report and a Review of the Literature. SAGE Open Medical Case Reports, 8, 2050313X-2091784X. https://doi.org/10.1177/2050313X20917846

[16] Sun, B.H., Yu, S.T., Ge, J.N. and Lei, S.-T. (2020) Primary Squamous Cell Carcinoma (PSCC) of the Thyroid: A Case Report and Review of the Literature. Gland Surgery, 9, 474-477. https://doi.org/10.21037/gs.2020.02.18

[17] Yang, S., Li, C., Shi, X., Ma, B., Xu, W., Jiang, H., et al. (2019) Primary Squamous Cell Carcinoma in the Thyroid Gland: A Population-Based Analysis Using the SEER Database. World Journal of Surgery, 43, 1249-1255. https://doi.org/10.1007/s00268-019-04906-2

[18] Struller, F., Senne, M., Falch, C., Kirschniak, A., Konigsrainer, A. and Muller, S. (2017) Primary Squamous Cell Carcinoma of the Thyroid: Case Report and Systematic Review of the Literature. International Journal of Surgery Case Reports, 37, 36-40. https://doi.org/10.1016/j.ijscr.2017.06.011

[19] Lichiardopol, C., Surlin, V., Foarfa, M.C., Ghiluşi, M.C. and Bondari, S. (2016) Primary Squamous Cell Carcinoma of the Thyroid: A Case Report. Romanian Journal of Morphology and Embryology, 57, 831-836.

[20] Dong, S., Song, X.S., Chen, G. and Liu, J. (2016) Mixed Primary Squamous Cell Carcinoma, Follicular Carcinoma, and Micropapillary Carcinoma of the Thyroid Gland: 
A Case Report. Auris Nasus Larynx, 43, 455-459.

https://doi.org/10.1016/j.anl.2015.10.011

[21] Kallel, S., Kallel, R., Ayadi, S. and Ghorbel, A. (2018) Primary Squamous Cell Carcinoma of the Thyroid Associated with Papillary Thyroid Carcinoma and Hashimoto's Thyroiditis. European Annals of Otorhinolaryngology, Head and Neck Diseases, 135, 291-293. https://doi.org/10.1016/j.anorl.2018.05.012

[22] Vita, R., Ieni, A., Tuccari, G. and Benvenga, S. (2018) The Increasing Prevalence of Chronic Lymphocytic Thyroiditis in Papillary Microcarcinoma. Reviews in Endocrine and Metabolic Disorders, 19, 301-309. https://doi.org/10.1007/s11154-018-9474-Z

[23] Uhliarova, B. and Hajtman, A. (2018) Hashimoto's Thyroiditis-An Independent Risk Factor for Papillary Carcinoma. Brazilian Journal of Otorhinolaryngology, 84, 729-735. https://doi.org/10.1016/j.bjorl.2017.08.012

[24] Fugazzola, L., Muzza, M., Pogliaghi, G. and Vitale, M. (2020) Intratumoral Genetic Heterogeneity in Papillary Thyroid Cancer: Occurrence and Clinical Significance. Cancers, 12, Article No. 383. https://doi.org/10.3390/cancers12020383

[25] Ho, A.S., Luu, M., Barrios, L., Chen, I., Melany, M., Ali, N., et al. (2020) Incidence and Mortality Risk Spectrum across Aggressive Variants of Papillary Thyroid Carcinoma. JAMA Oncology, 6, 706-713. https://doi.org/10.1001/jamaoncol.2019.6851

[26] Pradhan, D., Sharma, A. and Mohanty, S.K. (2015) Cribriform-Morular Variant of Papillary Thyroid Carcinoma. Pathology-Research and Practice, 211, 712-716.

https://doi.org/10.1016/j.prp.2015.04.011

[27] Torrez, M., Braunberger, R.C., Yilmaz, E. and Agarwal, S. (2020) Primary Squamous Cell Carcinoma of Thyroid with a Novel BRAF Mutation and High PDL-1 Expression: A Case Report with Treatment Implications and Review of Literature. Pathology-Research and Practice, 216, Article ID: 153146. https://doi.org/10.1016/j.prp.2020.153146

[28] Pekova, B., Sykorova, V., Dvorakova, S., Vaclavikova, E., Moravcova, J., Katra, R., et al. (2020) RET, NTRK, ALK, BRAF, and MET Fusions in a Large Cohort of Pediatric Papillary Thyroid Carcinomas. Thyroid, 30, 1771-1780. https://doi.org/10.1089/thy.2019.0802

[29] Pessoa-Pereira, D., Medeiros, M., Lima, V., da Silva Jr., J.C., de Oliveira Cerqueira, T.L., da Silva, I.C., et al. (2019) Association between BRAF (V600E) Mutation and Clinicopathological Features of Papillary Thyroid Carcinoma: A Brazilian SingleCentre Case Series. Archives of Endocrinology and Metabolism, 63, 97-106. https://doi.org/10.20945/2359-3997000000120

[30] Moulana, F.I., Priyani, A.A.H., de Silva, M.V.C. and Dassanayake, R.S. (2018) BRAFOncogene-Induced Senescence and the Role of Thyroid-Stimulating Hormone Signaling in the Progression of Papillary Thyroid Carcinoma. Hormones and Cancer, 9, 1-11. https://doi.org/10.1007/s12672-017-0315-4

[31] Xing, M., Alzahrani, A.S., Carson, K.A., Shong, Y.K., Kim, T.Y., Elisei, D.V.R., et al. (2015) Association between BRAFV600E Mutation and Recurrence of Papillary Thyroid Cancer. Journal of Clinical Oncology, 33, 42-50. https://doi.org/10.1200//CO.2014.56.8253

[32] Xing, M., Liu, R., Liu, X., Murugan, A.K., Zhu, G., ZeigerSara Pai, M.A., et al. (2014) $B R A F \mathrm{~V} 600 \mathrm{E}$ and TERT Promoter Mutations Cooperatively Identify the Most Aggressive Papillary Thyroid Cancer with Highest Recurrence. Journal of Clinical Oncology, 32, 2718-2726. https://doi.org/10.1200/JCO.2014.55.5094

[33] Haugen, B.R., Alexander, E.K., Bible, K.C., Doherty, G.M., Mandel, S.J., Nikiforov, Y.E., et al. (2016) 2015 American Thyroid Association Management Guidelines for 
Adult Patients with Thyroid Nodules and Differentiated Thyroid Cancer: The American Thyroid Association Guidelines Task Force on Thyroid Nodules and Differentiated Thyroid Cancer. Thyroid, 26, 1-133. https://doi.org/10.1089/thy.2015.0020

[34] Haugen, B.R. (2017) 2015 American Thyroid Association Management Guidelines for Adult Patients with Thyroid Nodules and Differentiated Thyroid Cancer: What Is New and What Has changed? Cancer, 123, 372-381.

https://doi.org/10.1002/cncr.30360

[35] Tiedje, V. and Fagin, J.A. (2020) Therapeutic Breakthroughs for Metastatic Thyroid Cancer. Nature Reviews Endocrinology, 16, 77-78.

https://doi.org/10.1038/s41574-019-0307-2

[36] Holoubek, S.A., Yan, H., Khokar, A.H., Kuchta, K.M., Winchester, D.J., Prinz, R.A., et al. (2020) Aggressive Variants of Papillary Thyroid Microcarcinoma Are Associated with High-Risk Features, but Not Decreased Survival. Surgery, 167, 19-27.

https://doi.org/10.1016/j.surg.2019.03.030

[37] Filetti, S., Durante, C., Hartl, D., Leboulleux, S., Locati, L.D., Newbold, K., et al. (2019) Thyroid Cancer: ESMO Clinical Practice Guidelines for Diagnosis, Treatment and Follow-Up. Annals of Oncology, 30, 1856-1883.

https://doi.org/10.1093/annonc/mdz400

[38] Limberg, J., Ullmann, T.M., Stefanova, D., Buicko, J.L., Finnerty, B.M., Zarnegar, R., et al. (2019) Does Aggressive Variant Histology Without Invasive Features Predict Overall Survival in Papillary Thyroid Cancer? A National Cancer Database Analysis. Annals of Surgery. https://doi.org/10.1097/SLA.0000000000003632

[39] Bongers, P.J., Kluijfhout, W.P., Verzijl, R., Lustgarten, M., Vermeer, M., Goldstein, D.P., et al. (2019) Papillary Thyroid Cancers with Focal Tall Cell Change Are as Aggressive as Tall Cell Variants and Should Not Be Considered as Low-Risk Disease. Annals of Surgical Oncology, 26, 2533-2539. https://doi.org/10.1245/s10434-019-07444-2

[40] Song, E., Jeon, M.J, Oh, H.S., Han, M., Lee, Y.-M., Kim, T.Y., et al. (2018) Do Aggressive Variants of Papillary Thyroid Carcinoma Have Worse Clinical Outcome than classic Papillary Thyroid Carcinoma? European Journal of Endocrinology, 179, 135-142. https://doi.org/10.1530/EJE-17-0991

[41] Zhu, J., Wang, X., Zhang, X., Li, P. and Hou, H. (2015) Clinicopathological Features of Recurrent Papillary Thyroid Cancer. Diagnostic Pathology, 10, Article No. 96. https://doi.org/10.1186/s13000-015-0346-5 